\title{
Pulmonary arterio-venous fistulae in hepatic cirrhosis
}

\author{
G KEREN, H BOICHIS, T S ZWAS, AND M FRAND \\ Departments of Paediatrics ' $C$ ' and Nuclear Medicine, Chaim Sheba Medical Centre, Tel-Hashomer, \\ and Sackler School of Medicine, Tel-Aviv University, Israel
}

SUMMARY Cyanosis, clubbing, and arterial oxygen desaturation may occur in patients with liver disease, and are attributed to the presence of pulmonary or peripheral arterio-venous shunting. Cardiac catheterisation and angiocardiography in a patient with a normal heart did not demonstrate the presence of abnormal arterio-venous anastomoses. Pulmonary shunting was proved when intravenous technetiumlabelled macroaggregated albumin, normally held up in capillary networks, was passed quickly through the lungs and immediately detected in high systemic blood flow organs. The opening of peripheral and pulmonary anastomoses in patients with liver disease may be owing to the presence of a vasodilatory substance such as ferritin, which was found to be abnormally increased in the patient's blood.

Blood oxygen desaturation may occur in patients with hepatic disease; it has been attributed to the existence of arterio-venous (A-V) shunts. Radiological confirmation of such $\mathrm{A}-\mathrm{V}$ shunts in the lungs is extremely difficult. We report on the demonstration of pulmonary A-V fistulae by means of pulmonary radioisotope scanning, using macroaggregated albumin particles.

\section{Case report}

A 16-year-old girl with cyanosis was referred. She had been well until age 13, when she contracted an acute illness associated with jaundice, diagnosed on clinical grounds as 'acute hepatitis'. Progressively increasing cyanosis and dyspnoea have been present since. On admission the patient was on the 10th centile for height and weight, had numerous spider naevi, and no telangiectasias of mucosae or fundi. Her pulse was $104 / \mathrm{min}$, her blood pressure $110 / 70$ $\mathrm{mmHg}$. She had severe central cyanosis, grade IV clubbing of the fingers and toes, and severe dyspnoea at rest. Examination of her heart and lungs was normal. Liver and spleen, both firm, were palpable $10 \mathrm{~cm}$ below the costal margin.

Laboratory investigations. These showed a haemoglobin level of $13.6 \mathrm{~g} / \mathrm{dl}$, with normal platelets, total white cell count, and differential count. Urine analysis was normal and there was no hyperaminoaciduria. Levels of serum calcium, phosphorus, alkaline phosphatase, cholesterol, alpha-1-antitrypsin, and ceruloplasmin were normal as was a sweat test. Levels of serum albumin were $28 \mathrm{~g} / \mathrm{l}$, globulin $41 \mathrm{~g} / 1$, AST 100-129 U/1, and prothrombin time 22 seconds $(30 \%)$. Serum ferritin level was 900 (normal < 140) ng/ml. Hepatitis B antibody and antigen could not be detected in the serum. Antinuclear factor was negative. Liver biopsy showed fibrotic bands with regenerative nodules devoid of central veins, with mononuclear inflammatory infiltration, and foci of liver cell necrosis. Chest $x$-ray film showed a normal cardiac silhouette with normal hilar markings, and the electrocardiogram was normal.

Arterial blood pH ranged from 7.42 to 7.45 . Arterial oxygen pressure was $45 \mathrm{mmHg}$, carbon dioxide pressure $22 \mathrm{mmHg}$, oxyhaemoglobin $82 \%$, and transcutaneous carbon dioxide pressure 14.7$17.5 \mathrm{mmol} / \mathrm{l}$. After breathing $100 \%$ oxygen for 20 minutes her arterial oxyhaemoglobin level rose from 83 to $97.5 \%$ but oxygen pressure from 45 to only 135 $\mathrm{mmHg}$, indicating a right-to-left shunt and amounting to $25 \%$ of the cardiac output. Echocardiography showed a normal heart and normal valvular function. On cardiac catheterisation normal pressures were recorded on the right side and in the pulmonary arteries, with no evidence of intracardiac shunting. The pulmonary vascular tree appeared normal on angiography with no anomalous venous drainage to the atria. Coeliac arteriography showed no anomalous visceral AV malformations.

In an attempt to prove the existence of an intrapulmonary right-to-left shunt, a perfusion lung scan using radioactive particles was performed. Immediately after the intravenous administration of $2 \mathrm{mC}$ of technetium-99m-macroaggregated albumin, 20-30 $\mu \mathrm{m}$ in diameter, a dynamic blood flow study was performed (Figure), in a sequence of 3 seconds a frame for one minute. Normal intracardiac blood flow was visualised with bilateral lung perfusion. In the second stage of the study, static views of the distribution of the radiotracer again showed a normal pulmonary perfusion and the simultaneous abnormal appearance of radioactivity over high 


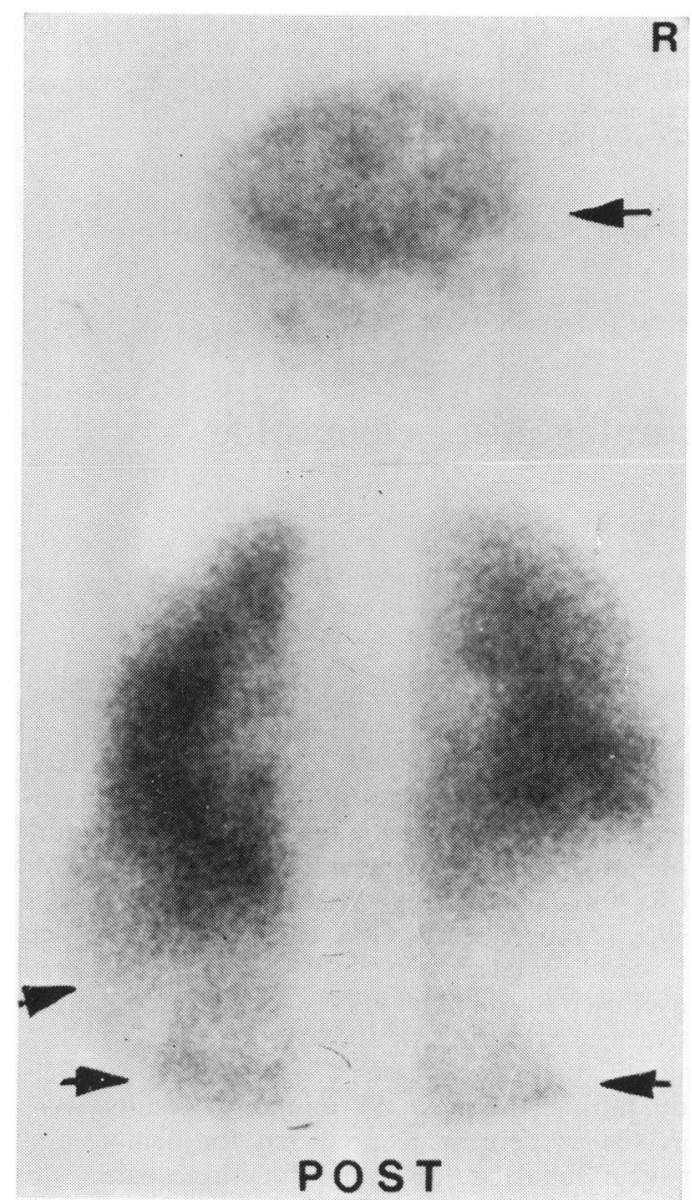

Figure Lung scan with technetium macroaggregated albumin, posterior view. Radioactivity appears over the head (upper arrow), the kidneys (bottom arrows), and the spleen (left middle arrow).

systemic blood flow organs such as the brain, the kidneys, and the spleen (Figure).

\section{Discussion}

Large congenital pulmonary A-V fistulae may be diagnosed on routine radiographic films of the chest. However, even in symptomatic cases, extensive investigations of the heart and lungs, including cardiac catherisation and angiocardiography, often fail to establish the diagnosis. ${ }^{1}$ Multiple pulmonary A-V fistulae are often of developmental or hereditary origin; however, they are also found in children with acute or chronic hepatic diseases, and may then be associated with cyanosis and digital clubbing. ${ }^{2-4}$

Arterial oxygen desaturation is found in many patients with cirrhosis. ${ }^{1-3}$ Such desaturation has been attributed to significant right-to-left shunting if it is not corrected completely by breathing $100 \%$ oxygen. Examinations at necropsy of patients with cirrhosis sometimes show small multiple A-V fistulae, even if pulmonary angiography had not shown them. ${ }^{45}$

Intravenous injection of radiolabelled macroaggregated albumin particles has recently been used to detect peripheral A-V fistulae. Normal capillaries are 8 to $15 \mu \mathrm{m}$ in diameter, and since the injected radiolabelled macroaggregated albumin particles measure 20 to $30 \mu \mathrm{m}$ in size, they are often wedged into the normal pulmonary capillaries during their first passage through the lungs, and do not pass into the systemic circulation. ${ }^{6}$ The rapid appearance of radioactivity over high systemic blood flow organssuch as the brain, the spleen, the thyroid, and kidneys-confirms the presence of a large left-to-right shunt. ${ }^{7}$

This fairly simple diagnostic procedure is recommended for investigating patients with liver disease and associated cyanosis and clubbing.

Right-to-left shunting in the lungs does not account for the persistence of cyanosis of lips and fingers, when normal arterial oxygen saturation is attained during $100 \%$ oxygen breathing, the so-called 'hepatogenic cyanosis' of Kravath et al. ${ }^{2}$ This phenomenon suggests that additional $A-V$ shunts may be open in the skin, decreasing the circulation of the peripheral capillaries. The association of hepatic disease and pulmonary and peripheral $\mathrm{A}-\mathrm{V}$ shunts has led to the hypothesis that the diseased liver releases a vasodilating substance, which opens preexisting, non-functioning precapillary anastomoses in the pulmonary and systemic circulations. ${ }^{8}$ It has been suggested that this substance is reduced ferritin, a substance released from hypoxic or injured liver, which is known to have a vasodilatory effect, and has been implicated in digital clubbing by virtue of its ability to open precapillary A-V anastomoses in fingers. ${ }^{9}$ The high ferritin blood levels found in our patient may fit this hypothesis.

\section{References}

1 Utzon F, Brandrup F. Pulmonary arteriovenous fistulas in children; a review with special reference to the disperse telangiectatic type, illustrated by report of a case. Acta Paediatr Scand 1973; 62: 422-32.

2 Kravath R E, Scarpelli E M, Bernstein J. Hepatogenic cyanosis; arteriovenous shunts in chronic active hepatitis. J Pediatr 1971; 78: 238-45.

${ }^{3}$ Silverman A, Cooper M D, Moller J H, Good R A. Syndrome of cyanosis, digital clubbing, and hepatic disease in siblings. J Pediatr 1968; 72: $70-80$. 
4 Rydell R, Hoff bauer F W. Multiple pulmonary arteriovenous fistulas in juvenile cirrhosis. Am J Med 1956; 21 : 450-60.

5 Murray J F, Dawson A M, Sherlock S. Circulatory changes in chronic liver disease. $A m \mathrm{~J} \mathrm{Med} \mathrm{1958;24:}$ 358-67.

6 Genovesi M G, Tierney D F, Taplin G V, Eisenberg H. An intravenous radionuclide method to evaluate hypoxemia caused by abnormal alveolar vessels. Am Rev Respir Dis 1976;114: 59-65.

7 Papanicolaou N, Treves S. Pulmonary scintigraphy in pediatrics. Semin Nucl Med 1980; 10: 259-85.
8 Tobin C E, Zariquiey M O. Arteriovenous shunts in the human lung. Proc Soc Exp Biol Med 1950; 75: 827-9.

9 Hall G H, Laidlaw C D. Further experimental evidence implicating reduced ferritin as a cause of digital clubbing. Clin Sci 1963; 24: 121-6.

Correspondence to Dr H Boichis, Department of Paediatrics 'C', Chaim Sheba Medical Centre, Tel-Hashomer 52621, Israel.

Received 10 September 1982

\title{
Bronchodilator effects of the $\mathrm{H}_{1}$ receptor antagonist-clemastine
}

\author{
R L HENRY, I G C HODGES, A D MILNER, AND G M STOKES \\ University Department of Child Health, University Hospital, Queen's Medical Centre, Nottingham
}

SUMmaRY We investigated the specific $\mathrm{H}_{1}$ receptor antagonist, clemastine, in 15 children with asthma. In the respiratory unit, clemastine was an effective bronchodilator but in a clinical trial we could not show any significant benefit compared with placebo.

Nogrady et al..$^{1}$ compared a nebulised solution of the specific $H_{1}$ receptor blocking antihistamine, clemastine, with salbutamol in adult asthmatics. Both drugs produced appreciable bronchodilatation, although the effect with clemastine was of slower onset but of more prolonged action. We have conflicting data on the bronchodilator properties of clemastine. ${ }^{2}{ }^{3}$ This trial was designed to assess its clinical role in a group of schoolchildren in whom asthma was poorly controlled on conventional therapy.

\section{Patients and methods}

Fifteen asthmatic children aged 7 years 9 months to 13 years 10 months (mean age $10 \frac{3}{4}$ years) took part in the study. They were receiving beclomethasone diproprionate ( 9 children), sodium cromoglycate ( 3 children), ketotifen ( 2 children), and oral steroids (one child). All of them were also receiving varying doses of salbutamol by inhalation.

The study design was a crossover trial with 6-week periods on placebo and clemastine, $200 \mu \mathrm{g}$ three times a day, delivered via a metered aerosol, in addition to the child's usual medications. Diary card records were kept of twice-daily peak expiratory flow rate (PEFR), total bronchodilator usage, and asthma symptom scores. The scoring system was a simple one with cough and wheeze graded separately by day and night. No symptoms scored zero and a rising score (up to 3 ) reflected clinical deterioration. The worst possible daily score was twelve.

At the end of each 6-week treatment period, each child visited the respiratory laboratory with instructions to omit drug therapy for at least 4 hours before attendance. Baseline values were obtained for PEFR, forced expiratory volume in 0.75 seconds $\left(\mathrm{FEV}_{0.75}\right),{ }^{4}$ and forced vital capacity, after which each child inhaled two puffs from the metered aerosol used in the previous 6 weeks. Lung function was measured during the next hour, after which a 6-minute run was performed and further readings made during the next 15 minutes. Results were expressed as percentage predicted for height ${ }^{5}$ and statistical analysis of lung function data was made by paired $t$ tests. The last 28 days of each treatment period was compared by Wilcoxon's rank sum tests.

\section{Results}

In the respiratory unit, clemastine resulted in significant improvement in PEFR and FEV $_{\mathbf{0 . 7 5}}$ compared with initial values (Table 1). It did not protect against exercised-induced asthma with mean

Table 1 PEFR and FEV $V_{0.75}$ expressed as percentage predicted after clemastine and placebo

\begin{tabular}{llllll}
\hline $\begin{array}{l}\text { Time } \\
(\min )\end{array}$ & \multicolumn{2}{l}{ Clemastine $($ mean $\pm I S D)$} & & \multicolumn{2}{l}{ Placebo $($ mean $\pm I S D)$} \\
\cline { 6 - 7 } \cline { 5 - 6 } & PEFR & $F E V_{0 \cdot 75}$ & & PEFR & $F E V_{0 \cdot 75}$ \\
\hline 0 & $76 \pm 15$ & $77 \pm 16$ & & $74 \pm 20$ & $72 \pm 23$ \\
5 & $78 \pm 14$ & $76 \pm 17$ & & $74 \pm 21$ & $71 \pm 24$ \\
15 & $81 \pm 12^{*}$ & $77 \pm 15$ & & $74 \pm 21$ & $71 \pm 23$ \\
30 & $81 \pm 12^{*}$ & $79 \pm 14$ & & $74 \pm 21$ & $72 \pm 23$ \\
60 & $84 \pm 13^{*}$ & $82 \pm 16 \dagger$ & & $75 \pm 22$ & $73 \pm 24$ \\
\hline
\end{tabular}

$\left.\begin{array}{l}\text { *P } 0.0: \\ +P 0.02\end{array}\right\}$ compared with initial. 